\title{
Hyperscaling Inequalities for Percolation
}

\author{
Hal Tasaki*
}

Physics Department, Princeton University, PO Box 708, Princeton, NJ 08544, USA

\begin{abstract}
A set of critical exponent inequalities for independent percolation which saturate under the hyperscaling hypothesis is proved. One of the consequences of the inequalities is the lower bound $d_{C} \geqq 6$ for the upper critical dimension. The proof is based on a rigorous version of the finite size scaling argument which extends easily to other systems such as Ising ferromagnets.
\end{abstract}

\section{Introduction}

In the present paper, we prove the following critical exponent inequalities for the independent percolation [1]

$$
\begin{gathered}
(d-2+\eta) \delta_{r} \geqq 2, \\
(d-2+\eta) v^{\prime} \geqq 2 \beta, \\
d v^{\prime} \geqq \gamma^{\prime}+2 \beta, \quad d v_{\max } \geqq \gamma+2 \beta, \\
d v \geqq 2 \Delta-\gamma, \\
d v^{\prime} \geqq \Delta^{\prime}+\beta, \quad d v_{\max } \geqq \Delta+\beta, \\
(d-2+\eta) \mu \delta \geqq 2, \\
d \mu \geqq 1+1 / \delta .
\end{gathered}
$$

These inequalities are of particular interest because of their close relation to the so-called hyperscaling hypothesis. If the hyperscaling hypothesis is valid, all the inequalities (1.1)-(1.7) become exact equalities.

Usually it is believed that the hyperscaling relations hold only in sufficiently low dimensions. As for independent percolation in two dimensions, Kesten [2] has recently proved almost all of the expected hyperscaling relations. However the validity of the hyperscaling hypothesis in dimensions higher than two is still wide

* Supported by NSF Grant PHY-85-15288-A01 
open, even on a heuristic level. It should be mentioned that the corresponding problem in the three dimensional Ising model has been an extremely difficult open problem for more than two decades [3].

On the other hand, in dimensions higher than the upper critical dimension, it is believed that the critical phenomena are governed by simple mean field theories. Therefore, in these dimensions, all the critical exponents assume dimension independent mean field values, and the hyperscaling relations are violated.

One of the most interesting features of our inequalities (1.1)-(1.7) is that they provide us with information about the upper critical dimension. More precisely, they are all inconsistent with the complete mean field type critical phenomena when the lattice dimension is smaller than six. This implies that the critical phenomena of percolation in dimensions two, three, four, and five are inevitably not mean fieldlike. In terms of the upper critical dimension $d_{C}$, our inequalities provide us with a rigorous lower bound $d_{C} \geqq 6$. Note that the lower bound $d_{C} \geqq 6$, as well as Aizenman and Newman's sufficient condition (triangle condition) for the mean field behavior [4], is consistent with the general belief $d_{C}=6$.

Though there have already been some critical exponent inequlaties which imply $d_{C} \geqq 4$ [5], and $d_{C} \geqq 6$ [6], our inequalities are the first ones that are believed to be sharp in sufficiently low dimensions. See $[7,8]$ and the references therein for other interesting critical exponent inequalities for percolation.

It should be mentioned that the first rigorous critical exponent inequality which saturates under the hyperscaling hypothesis was proved by Fisher [9] for Ising ferromagnets. Moreover the inequalities corresponding to (1.1)-(1.3), (1.6), (1.7) and special cases of (1.4), (1.5) have already been proved for certain ferromagnetic spin systems [9]. Since those proofs are based on the correlation inequalities and some specific features of the spin systems, none of them extend easily to percolation. ${ }^{1}$ Therefore instead of looking for possible extensions of the existing proofs, we here develop a new argument which is based on the finite size scaling idea [10]. It then turns out that the argument naturally leads us to the desired hyperscaling inequalities. Moreover our technique can be easily extended to other lattice systems such as Ising ferromagnets.

The organization of the present paper is as follows. In Sect. 2, we give precise definitions of the percolation system, some physical quantities, and various critical exponents. In Sect. 3, we discuss a consequence of our inequalities to the problem of the upper critical dimension. Then, in Sects. 4-7, we prove our critical exponent inequalities. In the Appendix, we extend the present method to Ising ferromagnets.

\section{Definitions}

For simplicity, we restrict ourselves to the neares neighbour bond percolation on the $d$-dimensional hypercubic lattice. However all of our results extend automatically to any translation invariant short range bond or site percolations.

\footnotetext{
${ }^{1}$ It is interesting that most of the existing proofs of the critical exponent inequalities which saturate under the scaling hypothesis (c.g., Fisher's $(2-\eta) v \geqq \gamma$ [9]) automatically extend to percolation without any modifications
} 
Let $Z^{d}$ be the $d$-dimensional hypercubic lattice whose elements $x, y, \ldots$ are called sites. We denote by 0 the origin of the lattice. A bond is an unordered pair $\{x, y\}$ of two sites satisfying $\|x-y\|_{2}=1$. In independent percolation, each bond in the lattice is occupied (respectively, unoccupied) independently with probability $p$ (respectively $1-p$ ). The occupation probability $p$ is our only model parameter. Let us denote by $\operatorname{Prob}_{p}(\ldots)$ and $\langle\ldots\rangle_{p}$ the probability and expectation value associated to the above process.

For a given configuration (i.e., occupation status of all the bonds in $Z^{d}$ ), we say that two sites $x, y$ are connected if there exists path of occupied bonds which connects $x$ and $y$. More precisely, $x$ and $y$ are connected if there exists a sequence of sites $\left\{x_{1}, \ldots, x_{n}\right\}$, where $x_{1}=x, x_{n}=y$, and each $\left\{x_{i}, x_{i+1}\right\}$ is an occupied bond. We also say that a site $x$ is connected to a set (of sites) $Y$ if there exists a site $y$ in $Y$ which is connected to $x$. Finally a cluster $C(x)$ is defined as a set of all the sites which are connected to $x,|C(x)|$ denotes the number of the sites in $C(x)$.

Now let us define some physical quantities of interest. The connectivity function $\tau_{p}(\ldots)$ and truncated connectivity function $\tau_{p}^{\prime}(\ldots)$ are

$$
\begin{gathered}
\tau_{p}\left(x_{1}, x_{2}, \ldots, x_{n}\right)=\operatorname{Prob}_{p}\left(C\left(x_{1}\right) \ni x_{2}, \ldots, x_{n}\right), \\
\tau_{p}^{\prime}\left(x_{1}, x_{2}, \ldots, x_{n}\right)=\operatorname{Prob}_{p}\left(C\left(x_{1}\right) \ni x_{2}, \ldots, x_{n},\left|C\left(x_{1}\right)\right|<\infty\right) .
\end{gathered}
$$

The mean (finite) cluster size $\chi(p)$ is

$$
\chi(\mathrm{p})=\langle|C(0)| X(|C(0)|<\infty)\rangle_{p}=\sum_{x} \tau_{p}^{\prime}(0, x)
$$

where $X(A)=1$ (or 0 ) when $A$ is true (or false). The correlation length $\xi(p)$ is

$$
\xi(p)=\inf \left\{\xi \mid \tau_{p}^{\prime}(0, x) \leqq e^{-|x| / \xi} \text { for any } x \in Z^{d}\right\} .
$$

Finally the order parameter $M(p)$ and its finite volume counterpart $M(p ; L)$ are

where

$$
\begin{gathered}
M(p)=\operatorname{Prob}_{p}(|C(0)|=\infty), \\
M(p ; L)=\operatorname{Prob}_{p}\left(0 \text { is connected to } \partial S_{L}\right),
\end{gathered}
$$

Throughout the present paper, we use the metric defined by

$$
|x|=\max \left\{\left|x_{1}\right|,\left|x_{2}\right|, \ldots,\left|x_{d}\right|\right\}=\|x\|_{\infty} \text {. }
$$

In the system with $d \geqq 2$, it is known [8] that there exists a critical probability $p_{c}$ $\left(0<p_{c}<1\right)$ which is characterized by

$$
\begin{array}{ll}
M(p)=0 & \text { if } \quad p<p_{c}, \\
M(p)>0 & \text { if } \quad p>p_{c} .
\end{array}
$$

It is also known that the mean cluster size $\chi(p)$ and the correlation length $\xi(p)$ diverge when $p$ approaches $p_{c}$ from below $[1,4]$. Note that if $p<p_{c}$, the truncated connectivity function $\tau_{p}^{\prime}(\ldots)$ is nothing but the connectivity function $\tau_{p}(\ldots)$, since the condition $\left|C\left(x_{1}\right)\right|<\infty$ is automatically satisfied. 
For the values of $p$ close to or equal to $p_{c}$, the percolation system is believed to exhibit various critical phenomena. More precisely, in this region many physical quantities are expected to show power law singularities characterized by the critical exponents. In the present paper, we do not assume the existence of the power law singularities, and introduce the critical exponents through the following formal definitions.

Let the relation $f(x) \lesssim x^{\lambda}$ as $x \searrow 0$ be an abbreviation for $f(x) \leqq s(x) x^{\lambda}$ for $x \geqq 0$ with a slowly varying function $s(x)$ (i.e., $\lim _{t \downarrow 0} s(t x) / s(t)=1$ for any $x>0$, e.g., $s(x)=$ const, $s(x)=|\ln x|)$. Then the critical exponents $\gamma, \gamma^{\prime}, \bar{\gamma}, \nu, v^{\prime}, \beta, \Delta_{n}, \Delta_{n}^{\prime}, \eta$, and $\delta_{r}$ are defined as the optimal constants satisfying the following relations. ${ }^{2}$

As $p \nearrow p_{c}$,

$$
\begin{gathered}
\chi(p) \gtrsim\left(p_{c}-p\right)^{-\gamma}, \\
\chi(p) \lesssim\left(p_{c}-p\right)^{-\bar{\gamma}}, \\
\xi(p) \lesssim\left(p_{c}-p\right)^{-v}, \\
\left\langle|C(0)|^{n-1}\right\rangle_{p} /\left\langle|C(0)|^{n-2}\right\rangle_{p} \gtrsim\left(p_{c}-p\right)^{-\Delta_{n}},
\end{gathered}
$$

as $p \searrow p_{c}$,

$$
\begin{gathered}
\chi(p) \gtrsim\left(p-p_{c}\right)^{-\gamma^{\prime}}, \\
\xi(p) \lesssim\left(p-p_{c}\right)^{-v^{\prime}}, \\
M(p) \lesssim\left(p-p_{c}\right)^{\beta}, \\
\left\langle|C(0)|^{n-1} X(|C(0)|<\infty)\right\rangle_{p} /\left\langle|C(0)|^{n-2} X(|C(0)|<\infty\rangle_{p} \gtrsim\left(p-p_{c}\right)^{-\Delta_{n}^{\prime}},\right.
\end{gathered}
$$

at $p=p_{c}$,

$$
\begin{gathered}
\tau_{p_{c}}(x, y) \gtrsim|x-y|^{-(d-2+\eta)}, \\
M\left(p_{c} ; L\right) \lesssim L^{-1 / \delta_{r}} .
\end{gathered}
$$

Here the specific choice of upper or lower bound is merely from technical considerations. Usually one believes that the above relations with $\lesssim$ or $\gtrsim$ replaced by $\sim\left(f \sim x^{\lambda}\right.$ means $f \lesssim x^{\lambda}$ and $\left.f \gtrsim x^{\lambda}\right)$ are valid. (Thus, in particular, we have $\left.\gamma=\bar{\gamma}_{\text {. }}\right)$ See Sect. 7 for the definitions of the other critical exponents.

\section{Critical Dimension}

In a suitable mean field theory (e.g., Cayley tree model, $d \rightarrow \infty$ limit) for percolation, one can easily calculate the critical behavior of many quantities explicitly. Then we find that many quantities exhibit strict power law behaviors [the relations like (2.8)-(2.16) with $\lesssim$ or $\gtrsim$ replaced by $\sim$ ] with the critical exponents $\gamma=\gamma^{\prime}=1$, $v=v^{\prime}=1 / 2, \beta=1, \Delta_{n}=\Delta_{n}^{\prime}=2, \eta=0, \delta_{r}=1 / 2, \mu=1 / 4$, and $\delta=2$. (See Sect. 7 for the definitions of the exponents $\mu$ and $\delta$.)

\footnotetext{
${ }^{2}$ Note that our definition of the gap exponent $\Delta_{n}$ differs from that in some articles (such as [2]) in percolation. But ours is a natural extension of the standard definition in the spin systems
} 
Let us substitute these mean field values of the critical exponents into our inequalities (1.1)-(1.7). Then we find that each of the inequalities leads us to a single inequality $d \geqq 6$. This implies that the complete mean field type critical phenomea are inconsistent with our critical exponent inequalities (and with their counterparts for the original physical quantities such as $\xi, \chi$, and $M$ ) if the lattice dimension is smaller than six. In other words, the upper critical dimension $d_{c}$ of the translation invariant short range independent percolation cannot be less than six!

Note that the lower bound $d_{c} \geqq 6$ was already mentioned in [6], where the bound was concluded from the other critical exponent inequalities (which were however not optimal as the present ones).

\section{Basic Inequalities}

In the present section, we prove our most basic inequalities (1.1) and (1.2). Although the derivation of these inequalities is rather elementary and straightforward, it contains some of the essential ideas of the present rigorous finite size scaling approach.

Proposition 4.1. For arbitrary positive integer $L$ and $x \in Z^{d}$ with $|x|=L$, we have

$$
\tau_{p}(0, x) \leqq M(p ; L)^{2} .
$$

From the above inequality with $p=p_{c}$ and the definition of the critical exponents $\eta$ and $\delta_{r}$, we immediately get

Corollary 4.1. The critical exponents $\eta$ and $\delta_{r}$ satisfy

$$
(d-2+\eta) \delta_{r} \geqq 2 .
$$

Proof of Proposition. Observe that when 0 and $x$ are connected, each of them must be connected to some site at a distance $[L / 2]$ of each (Fig. 1). Since the latter two events take place in the two separated regions in the lattice, we get the desired bound (4.1).

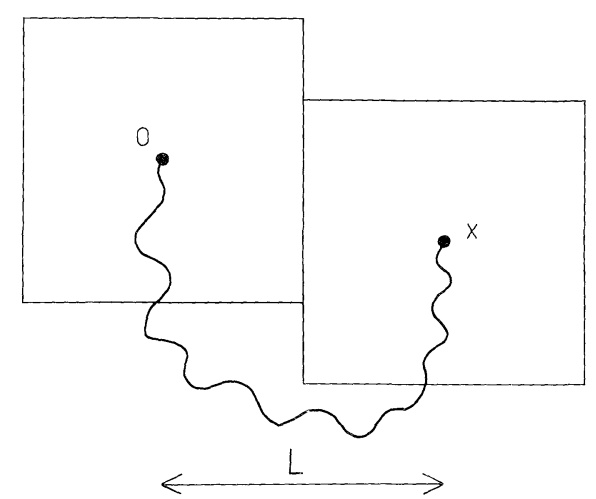

Fig. 1. The event that 0 and $x$ are connected 


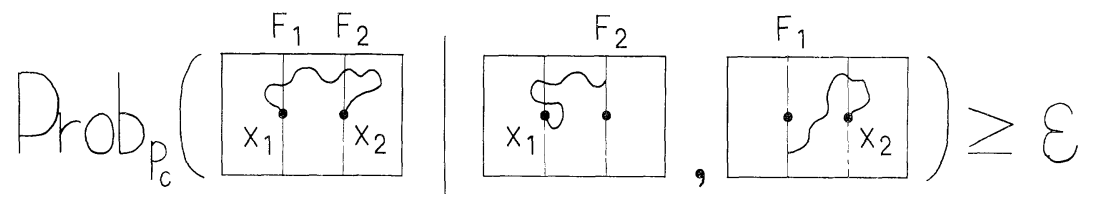

Fig. 2. A sufficient condition for the hyperscaling relation $(d-2+\eta) \delta_{r}=2$

Remark. Let $L=4 n$ with a positive integer $n$. We consider the percolation system in a finite lattice $\left\{y\left|(-3 / 4) L \leqq y_{1} \leqq(3 / 4) L,\right| y_{i} \mid \leqq L / 2(i=2, \ldots, d)\right\}$. We define (see Fig. 2)

$$
\begin{aligned}
& F_{1}=\left\{y\left|y_{1}=-L / 4,\right| y_{i} \mid \leqq L / 2(i=2, \ldots, d)\right\}, \\
& F_{2}=\left\{y\left|y_{1}=L / 4,\right| y_{i} \mid \leqq L / 2(i=2, \ldots, d)\right\},
\end{aligned}
$$

$x_{1}=(-L / 4,0,0, \ldots)$, and $x_{2}=(L / 4,0,0, \ldots)$. Consider the following condition (see Fig. 2):

$\operatorname{Prob}_{p_{\mathrm{c}}}\left(x_{1}\right.$ and $x_{2}$ are connected $\mid x_{1}$ is connected to $F_{2}, x_{2}$ is connected to $\left.F_{1}\right) \geqq \varepsilon$

holds with $\varepsilon>0$ uniformly in $L$. Here $\operatorname{Prob}(A \mid B)=\operatorname{Prob}(A, B) / \operatorname{Prob}(B)$ denotes the conditional probability. Note that the above probability is nothing but the intersection probability of two large clusters at $p_{c}$. Then it is not difficult to show that the above condition [along with (4.1)] implies the relation $\tau_{p_{c}}(0,(L, 0,0, \ldots))$ $\sim M\left(p_{c} ; L\right)^{2}$ which reduces to a hyperscaling relation $(d-2+\eta) \delta_{r}=2$. However we do not know any methods of proving (or disproving) the above condition in the dimensions higher than two.

In order to eliminate the rather unfamiliar exponent $\delta_{r}$ from (4.1), and to get our next inequality (1.2), we will make use of the idea of the heuristic finite size scaling theory [10].

In the finite size scaling theory, it is argued that the finite size order parameter $M\left(p_{c} ; L\right)$ behaves almost similarly to the full order parameter $M(p)$ evaluated at the value of $p\left(p>p_{c}\right)$ which satisfies $\xi(p)=L$. Although it is very hard to justify this conjecture in general (but see [2] for the results in two dimensions), the following weaker version can be proved very easily.

Lemma 4.1. For arbitrary $p>p_{c}$ with $\xi(p) \geqq C_{1}$, we have

$$
M(p) \leqq M(p ; L) \leqq 2 M(p)
$$

when $L=2 d \xi(p)|\ln \xi(p)|$. Here $C_{1}$ is a constant which depends only on the dimension.

Here the choice of constants $2 d$ and 2 are rather arbitrary. In two dimensions, Nguyen [11] has proved the above bound without (unwanted) $\ln \xi$ factor.

Proof. The first inequality is trivial since whenever the origin is connected to infinity, it must be connected to $\partial S_{L}$. Let $B_{L}$ be the event that the origin is connected to $\partial S_{L}$. 
To prove the second inequality, note that

$$
\begin{aligned}
M(p ; L) & =\operatorname{Prob}_{p}\left(B_{L}\right) \\
& =\left\{1-\operatorname{Prob}_{p}\left(B_{L},|C(0)|<\infty\right) / \operatorname{Prob}_{p}\left(B_{L}\right)\right\}^{-1} \operatorname{Prob}_{p}\left(B_{L},|C(0)|=\infty\right) \\
& \leqq\left\{1-\operatorname{Prob}_{p}\left(B_{L},|C(0)|<\infty\right) / \operatorname{Prob}_{p}\left(B_{L}\right)\right\}^{-1} M(p) .
\end{aligned}
$$

Let us construct an upper bound for the prefactor. Recall that Simon's argument [12] combined with the percolation version of Simon's inequality [4] implies that

$$
\sum_{x \in \hat{\partial} S_{L}} \tau_{p}(0, x) \geqq 1 \quad \text { if } \quad p \geqq p_{c} .
$$

Therefore one can find $x$ in $\partial S_{L}$ with the property $\tau_{p}(0, x) \geqq\left(2 d L^{d-1}\right)^{-1}$. Thus we get

$$
\operatorname{Prob}_{p}\left(B_{L}\right) \geqq \tau_{p}(0, x) \geqq\left(2 d L^{d-1}\right)^{-1} .
$$

On the other hand observe that

$$
\operatorname{Prob}_{p}\left(B_{L},|C(0)|<\infty\right) \leqq \sum_{x \in \partial S_{L}} \tau_{p}^{\prime}(0, x) \leqq 2 d L^{d-1} e^{-L / \xi(p)},
$$

where we used the defintion (2.4) of $\xi(p)$. Substituting (4.6), (4.7) into (4.4), we get

$$
M(p ; L) \leqq\left\{1-\left(2 d L^{d-1}\right)^{2} e^{-L / \xi(p)}\right\}^{-1} M(p) .
$$

For $L=2 d \xi(p)|\ln \xi(p)|$ and sufficiently large $\xi(p)$, the right-hand side of the above bound is bounded by $2 M(p)$.

Let us define the quantity $p(L)$ by the following formula:

$$
p(L)=\inf \left\{p\left|p>p_{c}, 2 d \xi(p)\right| \ln \xi(p) \mid \leqq L\right\} .
$$

Note that if $\zeta(p)$ is a monotone continuous function (as is expected), $p(L)$ is nothing but the inverse function of $2 d \xi(p)|\ln \xi(p)|$.

Proposition 4.2. For arbitrary $L \geqq C_{1}$ and $x$ with $|x|=L$, we have

$$
\tau_{p_{c}}(0, x) \leqq 4 M(p(L))^{2},
$$

provided that $\xi(p) \nearrow$ To as $p \bigsqcup_{\downarrow} p_{c}$.

Proof. Since $M(p ; L) \geqq M\left(p_{c} ; L\right)$ for $p \geqq p_{c}$, (4.9) follows immediately from Proposition 4.1 and Lemma 4.1 .

If we note that the relation $\xi(p) \lesssim\left(p-p_{c}\right)^{-v^{\prime}}$ implies $p(L)-p_{c} \lesssim\left(p-p_{c}\right)^{-1 / v^{\prime}}$, we get

Corollary $\AA_{0.2}$. Whenever $\xi(p) \nearrow \infty$ as $p \backslash p_{c}$, the critical exponents $\eta, v^{\prime}$, and $\beta$ satisfy

$$
(d-2+\eta) v^{\prime} \geqq 2 \beta \text {. }
$$

Remark. Combining the consequence of Simon's argument (4.5) and the present idea, we get the following strict lower bound for the finite size order parameter $M(p ; L)$

$$
M(p ; L) \geqq\left(2^{d} d\right)^{-1 / 2} L^{-(d-1) / 2} \quad \text { if } \quad p \geqq p_{c}
$$


This bound, which was first proved by Aizenman [13], is a generalization of van den Berg and Kesten's result in two dimensions [14].

\section{Inequalities for $v, \gamma$, and $\beta$}

In the present section, we prove the inequalities (1.3). We think that these are the best critical exponent inequalities among those proved in the present paper, since they only include simple and standard critical exponents which are also "approach exponents". Here the "approach exponents" means the critical exponents defined through the singular behavior which takes place when the system approaches its critical point.

Proposition 5.1. For arbitrary $p>p_{c}$ with $\xi(p) \geqq C_{2}$, we have

$$
\chi(p) \leqq C_{3} \sum_{L=\{(d / 2) \xi(p)|\ln \xi(p)|\}^{1 / d}}^{2 d \xi(p)|\ln \xi(p)|} L^{d-1} M(p(L))^{2},
$$

where $C_{2}$ and $C_{3}$ are constants which depend only on the dimension. $(p(L)$ is defined by (4.9).)

Let us again assume that $\xi(p) \nearrow \infty$ as $p \backslash p_{c}$. Then as $p$ approaches $p_{c}$, any $p(L)$ in the summation in (5.1) also approaches $p_{c}$. Then we can substitute the critical behavior of the quantities into (5.1) to get

$$
\left(p-p_{c}\right)^{-\gamma^{\prime}} \lesssim \sum_{L=1}^{2 d \xi(p)|\ln \xi(p)|} L^{d-1} L^{-2 \beta / v^{\prime}} \lesssim\left(p-p_{c}\right)^{-d v^{\prime}+2 \beta} .
$$

This immediately implies

Corollary 5.1. Whenever $\xi(p) \nearrow \infty$ as $p \searrow p_{c}$, the critical exponents $v^{\prime}, \gamma^{\prime}$, and $\beta$ satisfy

$$
d v^{\prime} \geqq \gamma^{\prime}+2 \beta \text {. }
$$

Proof of Proposition. Let us bound $\chi(p)$ by the following three terms:

$$
\chi(p)=\sum_{x} \tau_{p}^{\prime}(0, x) \leqq \sum_{x ;|x| \leqq L_{1}} 1+\sum_{x ; L_{1}<|x| \leqq L_{2}} \tau_{p}(0, x)+\sum_{x: L_{2}<|x|} \tau_{p}^{\prime}(0, x),
$$

where $L_{1}=\{(d / 2) \xi(p)|\ln \xi(p)|\}^{1 / d}, L_{2}=2 d \xi(p)|\ln \xi(p)|$. Here we have used the trivial inequalities $\tau_{p}^{\prime}(0, x) \leqq \tau_{p}(0, x) \leqq 1$. Using the bound (4.5) (which is a consequence of Simon's argument), the first and second terms in (5.2) can be related as

$$
\sum_{x:|x| \leqq L_{1}} 1=d \xi(p)|\ln \xi(p)| \leqq \sum_{x ; L_{1}<|x| \leqq L_{2}} \tau_{p}(0, x)
$$

From the definition of $\xi(p)$, the third term is bounded as

$$
\sum_{x ; L_{2}<|x|} \tau_{p}^{\prime}(0, x) \leqq \sum_{L>L_{2}} 2 d L^{d} e^{-L / \xi(p)} \leqq \xi(p)^{-a}
$$

with $a>0$ and $\xi(p) \geqq C^{\prime}$, where $C^{\prime}$ is a sufficiently large constant. Combining these 
bounds together we get an upper bound

$$
\chi(p) \leqq \text { const } \sum_{x ; L_{1}<|x| \leqq L_{2}} \tau_{p}(0, x),
$$

provided that $\xi(p) \geqq C_{2}$, where $C_{2}$ is a constant which depends only on the dimension. Now noting that $p(L) \geqq p$ for $L \leqq L_{2}$, we can repeat the arguments in the previous section to get

$$
\tau_{p}(0, x) \leqq 4 M(p(L))^{2} \text { for } x \text { with }|x|=L,
$$

which leads us to the desired bound (5.1) when summed over $L$.

By a slight modification of the above proof, we can also show the following result for the behaviour of the mean cluster size in the low density region.

Proposition 5.2. For arbitrary $p<p_{c}$ with $\xi(p) \geqq C_{2}$, we have

$$
\chi(p) \leqq C_{3} \sum_{L=\{(d / 2) \xi(p)|\ln \xi(p)|\} 1 / d}^{2 d \xi(p)|\ln \xi(p)|} L^{d-1} M(p(L))^{2},
$$

provided that $\xi(p) \nearrow \infty$ as $p \searrow p_{c}$.

Note that $\xi(p) \nearrow \infty$ as $p \nearrow p_{c}$ is known rigorously $[1,4]$.

As before we get the following critical exponent inequality from (5.3):

$$
d v \geqq \gamma+2 \beta\left(v / v^{\prime}\right)
$$

If $v / v^{\prime} \geqq 1$, (5.4) implies $d v \geqq \gamma+2 \beta$. On the other hand if $v^{\prime} / v \geqq 1$, we multiply (5.4) by $v^{\prime} / v$ to find $d v^{\prime} \geqq \gamma\left(v^{\prime} / v\right)+2 \beta \geqq \gamma+2 \beta$. Therefore we get

Corollary 5.2. Whenever $\xi(p) \nearrow \infty$ as $p \downarrow p_{c}$, the critical exponents $v_{\max }=\max \left(v, v^{\prime}\right)$, $\gamma$ and $\beta$ satisfy

$$
d v_{\max } \geqq \gamma+2 \beta
$$

\section{Inequalities for Gap Exponents}

In the present section, we prove the inequalities (1.4), (1.5) for the gap exponents $\Delta_{n}$ and $\Delta_{n}^{\prime}$.

First let us state a simple inequality which will be used in the following proofs. (The inequality was also noted by Nguyen [4].)

Lemma 6.1. For arbitrary $p$ and $n \geqq 3$, we have

$$
\begin{aligned}
& \left\langle|C(0)|^{n-1} X(|C(0)|<\infty)\right\rangle_{p} /\left\langle|C(0)|^{n-2} X(|C(0)|<\infty)\right\rangle_{p} \\
& \leqq\left\langle|C(0)|^{n} X(|C(0)|<\infty)\right\rangle_{p} /\left\langle|C(0)|^{n-1} X(|C(0)|<\infty)\right\rangle_{p} .
\end{aligned}
$$

Proof. By the Schwarz inequality we get

$$
\begin{aligned}
& \left\langle|C(0)|^{n-1} X(|C(0)|<\infty)\right\rangle_{p}=\left\langle|C(0)|^{n / 2}|C(0)|^{(n-2) / 2} X(|C(0)|<\infty)\right\rangle_{p} \\
& \leqq\left(\left\langle|C(0)|^{n} X(|C(0)|<\infty)\right\rangle_{p}\left\langle|C(0)|^{n-2} X(|C(0)|<\infty)\right\rangle_{p}\right)^{1 / 2},
\end{aligned}
$$

which is nothing but (6.1). 
Remark. Note that if we assume the power law behaviors

$$
\begin{gathered}
\left\langle|C(0)|^{n-1}\right\rangle_{p} /\left\langle|C(0)|^{n-2}\right\rangle_{p} \sim\left(p_{c}-p\right)^{-\Delta_{n}} \text { as } p T^{\prime} p_{c}, \\
\left\langle|C(0)|^{n-1} X(|C(0)|<\infty)\right\rangle_{p} /\left\langle|C(0)|^{n-2} X(|C(0)|<\infty)\right\rangle_{p} \sim\left(p-p_{c}\right)^{-\Delta_{n}^{\prime}} \text { as } p \downarrow p_{c},
\end{gathered}
$$

the inequality (6.1) implies the critical exponent inequalities

$$
\Delta_{n} \leqq \Delta_{n+1}, \quad \Delta_{n}^{\prime} \leqq \Delta_{n+1}^{\prime} .
$$

These inequalities become equalities under the scaling hypothesis. Therefore they are believed to be sharp in any dimensions.

The following inequality (6.3) is essentially a simple consequence of the van den Berg, Kesten inequality [14]. But it leads us to our first hyperscaling inequality for the gap exponents.

Proposition 6.1. For arbitrary $n \geqq 3$ and $p<p_{c}$ with $\xi(p) \geqq C_{4}$, we have

$$
\left(\left\langle|C(0)|^{n-1}\right\rangle_{p} /\left\langle|C(0)|^{n-2}\right\rangle_{p}\right)^{2} \leqq C_{5}(\xi(p)|\ln \xi(p)|)^{d} \chi(p),
$$

where $C_{4}$ and $C_{5}$ are constants which depend only on the dimension.

Since we know rigorously that $\xi(p)$ diverges when $p$ approaches $p_{c}$ from below, this leads us to

Corollary 6.1. The critical exponents $v, \Delta_{n}(n \geqq 3)$ and $\bar{\gamma}$ satisfy

$$
d v \geqq 2 \Delta_{n}-\bar{\gamma} .
$$

In order to prove the proposition, we have to state a simple geometric lemma. Let $\left\{x_{1}, \ldots, x_{n}\right\}$ be an arbitrary set of sites (which need not to be distinct). For a configuration (i.e., occupation status of all the bonds) which satisfies $C\left(x_{1}\right) \ni x_{2}, \ldots, x_{n}$, a pair of sites $\left\{y, y^{\prime}\right\} \subset\left\{x_{1}, \ldots, x_{n}\right\}$ is called a separable pair if there exists a path of occupied bonds $\omega$ which i) connects $y$ and $y^{\prime}$, and ii) all the sites in $\left\{x_{1}, \ldots, x_{n}\right\} \backslash\left\{y, y^{\prime}\right\}$ remain connected with each other when we remove all the bonds on $\omega$ (see Fig. 3).

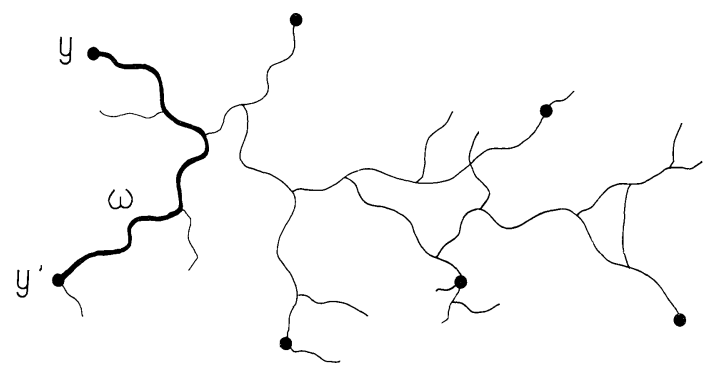

Fig. 3. A separabel pair $\left\{y, y^{\prime}\right\}$ and a path $\omega$ 


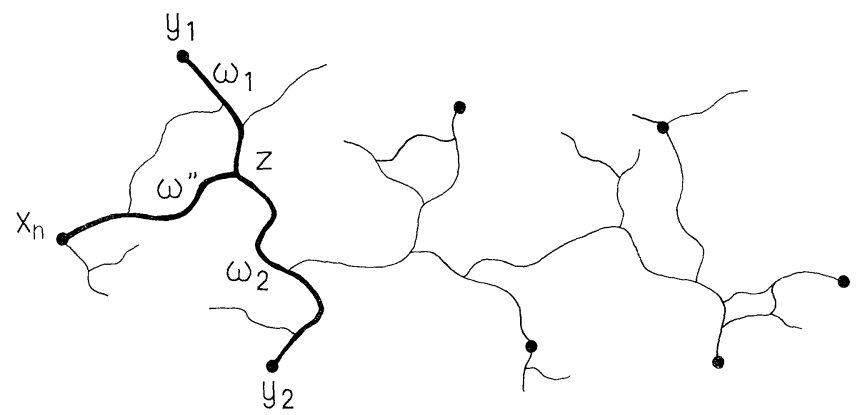

Fig. $4\left\{x_{n}, y_{1}\right\}$ becomes a new separable pair

Lemma 6.2. For an arbitrary set of sites $\left\{x_{1}, \ldots, x_{n}\right\}$ and an arbitrary configuration where $C\left(x_{1}\right) \ni x_{2}, \ldots, x_{n}$, there exists at least one separable pair $\left\{y, y^{\prime}\right\}$ $\subset\left\{x_{1}, \ldots, x_{n}\right\}$.

Proof. Since the statement is trivial when $n=2$, we proceed by induction. Let $n>2$. First, by omitting the site $x_{n}$, we may apply the lemma for $n-1$ to find a separable pair $\left\{y_{1}, y_{2}\right\} \subset\left\{x_{1}, \ldots, x_{n-1}\right\}$ and a path $\omega^{\prime}$ which connects $y_{1}$ and $y_{2}$. When we remove all the bonds on $\omega^{\prime}$ from the configuration, the site $x_{n}$ may be i) still connected to $\left\{x_{1}, \ldots, x_{n-1}\right\} \backslash\left\{y_{1}, y_{2}\right\}$ or ii) disconnected from $\left\{x_{1}, \ldots, x_{n-1}\right\} \backslash\left\{y_{1}, y_{2}\right\}$. If $\left.\mathrm{i}\right)$ is the case, we are done by taking $\left\{y, y^{\prime}\right\}=\left\{y_{1}, y_{2}\right\}$. Let us assume ii). Then we can find a path of occupied bonds $\omega^{\prime \prime}$ (with $\omega^{\prime} \cap \omega^{\prime \prime}=\phi$ ) which connects $x_{n}$ to a site $z$ in $\omega^{\prime}$. Decompose $\omega^{\prime}$ as $\omega_{1} \cup \omega_{2}$ by cutting it at $z$. (We assume $\omega_{i} \ni y_{i}$.) Observe that the set $\left\{x_{1}, \ldots, x_{n-1}\right\} \backslash\left\{y_{1}, y_{2}\right\}$ must be connected to at least one of $\omega_{1}$ or $\omega_{2}$ (which we call $\omega_{i}$ ) without using the bonds in $\omega^{\prime \prime}$. (If this is not the case, it contradicts with our assumption ii) (see Fig. 4). Then $\left\{y, y^{\prime}\right\}=\left\{x_{n}, y_{j}\right\}(i \neq j)$ is a separable pair $\left\{x_{1}, \ldots, x_{n}\right\}$ with the corresponding path $\omega=\omega^{\prime \prime} \cup \omega_{j}$.

Proof of Proposition. From the expression $|C(0)|=\sum_{x} X(C(0) \ni x)$ and the translation invariance, we have

$$
\left\langle|C(0)|^{n}\right\rangle_{p}=\sum_{x_{2}, \ldots, x_{n+1}} \tau_{p}\left(x_{1}, x_{2}, \ldots, x_{n+1}\right) .
$$

Let $D_{\max }$ be an abbreviation for $\max \left\{\left|x_{2}-x_{1}\right|,\left|x_{3}-x_{1}\right|,\left|x_{4}-x_{1}\right|, \ldots,\left|x_{n+1}-x_{1}\right|\right\}$. Then, since $\tau_{p}\left(x_{1}, \ldots, x_{n+1}\right) \leqq \tau_{p}\left(x_{1}, x_{i}\right)$ for any $i$, we get the following finite volume estimate for $\left\langle|C(0)|^{n}\right\rangle_{p}$.

$$
\begin{gathered}
\left\langle|C(0)|^{n}\right\rangle_{p}-\sum_{\substack{x_{2}, \ldots, x_{n+1} \\
D_{\max } \leqq C_{6} \xi(p)|\ln \xi(p)|}} \tau_{p}\left(x_{1}, x_{2}, \ldots, x_{n+1}\right) \\
\leqq \\
\sum_{L=C_{6} \xi(p)|\ln \xi(p)|}^{\infty} \sum_{\substack{x_{2}, \ldots, x_{n+1} \\
D_{\max }=L}} \tau_{p}\left(x_{1}, \ldots, x_{n+1}\right) \\
\leqq \\
\sum_{L=C_{6} \xi(p)|\ln \xi(p)|}^{\infty} n(2 L)^{n d-1} e^{-L / \xi(p)} \leqq \xi(p)^{-a^{\prime}} .
\end{gathered}
$$


Here $a^{\prime}>0$, and the final inequality is valid when $\xi(p) \geqq C^{\prime \prime}$ with sufficiently large $C^{\prime \prime}$. Now by applying Lemma 6.2 , we immediately get

$$
\begin{gathered}
\sum_{x_{2} \ldots, x_{n+1}, D_{\max } \leqq C_{6} \xi(p)|\ln \xi(p)|} \tau_{p}\left(x_{1}, \ldots, x_{n+1}\right) \\
\leqq \\
\sum_{\substack{\text { pairs } \\
\{i, j\} \subset\{1, \ldots, n+1\}}} \sum_{x_{2}, \ldots, x_{n+1}, D_{\max } \leqq C_{6} \xi(p)|\ln \xi(p)|}
\end{gathered}
$$

$\operatorname{Prob}_{p}\left\{\left(x_{1}, \ldots, \hat{x}_{i}, \ldots, \hat{x}_{j}, \ldots, x_{n+1}\right.\right.$ are connected $) \circ\left(x_{i}, x_{j}\right.$ are connected $\left.)\right\}$,

where $\hat{x}_{i}$ denote omitted sites. Here $A \circ B$ stands for the event that two events $A$ and $B$ occur disjointly [14]. For positive events $A, B$, van den Berg and Kesten [14] have proved that $\operatorname{Prob}_{p}(A \circ B) \leqq \operatorname{Prob}_{p}(A) \operatorname{Prob}_{p}(B)$. Therefore the right-hand side of the above inequality is bounded as

$$
\begin{aligned}
& \leqq \sum_{\text {pairs }\{l, j\} \subset\{1, \ldots, n+1\}} \sum_{\substack{x_{2}, \ldots, x_{n+1} \\
D_{\max } \leqq C_{6} \xi(p)|\ln \xi(p)|}} \tau_{p}\left(x_{1}, \ldots, \hat{x}_{i}, \ldots, \hat{x}_{j}, \ldots, x_{n+1}\right) \tau_{p}\left(x_{i}, x_{j}\right) \\
& \leqq\{n(n+1) / 2\}\left\langle|C(0)|^{n-2}\right\rangle_{p} \chi(p)\left(2 C_{6} \xi(p)|\ln \xi(p)|\right)^{d} .
\end{aligned}
$$

Combining this result with (6.1), we get the desired inequality (6.3).

Finally we state inequalities which follow from a combination of the methods in the present and previous sections.

Proposition 6.2. For arbitrary $n \geqq 3$ and $p>p_{c}$ with $\xi(p) \geqq C_{7}$, we have

$$
\begin{aligned}
& \left(\left\langle|C(0)|^{n-1} X(|C(0)|<\infty)\right\rangle_{p} /\left\langle|C(0)|^{n-2} X(|C(0)|<\infty)\right\rangle_{p}\right)^{2} \\
& \leqq C_{8} \sum_{L=\left\{\left(C_{6} / 2\right) \xi(p)|\ln \xi(p)|\right\}^{1 / d}}^{C_{6} \xi(p)|\ln \xi(p)|} L^{d-1} M\left(p^{\prime}(L)\right)^{2},
\end{aligned}
$$

where $C_{6}, C_{7}$ and $C_{8}$ are constants which depend only on the dimension. Also assume that $\xi(p) \nearrow \infty$ as $p \backslash p_{c}$. Then for arbitrary $n \geqq 3$ and $p<p_{c}$ with $\xi(p) \geqq C_{7}$, we have

$$
\left.\left.\left(\left\langle|C(0)|^{n-1}\right)\right\rangle_{p} /\left\langle|C(0)|^{n-2}\right)\right\rangle_{p}\right)^{2} \leqq C_{8} \sum_{L=\left\{\left(C_{6} / 2\right) \xi(p)|\ln \xi(p)|\right\}^{1 / d}}^{C_{6} \xi(p)|\ln \xi(p)|} L^{d-1} M\left(p^{\prime}(L)\right)^{2} .
$$

Here $p^{\prime}(L)$ is defined by $p^{\prime}(L)=\inf \left\{p\left|C_{6} \xi(p)\right| \ln \xi(p) \mid \leqq L\right\}$.

As in the previous section, these inequalities imply the following critical exponent inequalities.

Corollary 6.2. Whenever $\xi(p) \nearrow \infty$ as $p \backslash p_{c}$, the critical exponents $v^{\prime}, v_{\max }$ $=\max \left\{v, v^{\prime}\right\}, \Delta_{n}, \Delta_{n}^{\prime}(n \geqq 3)$ and $\beta$ satisfy

$$
\begin{gathered}
d v^{\prime} \geqq \Delta_{n}^{\prime}+\beta, \\
d v_{\max } \geqq \Delta_{n}+\beta .
\end{gathered}
$$

Proof of Proposition. The proof is almost a repetition of those of Propositions 6.1, 5.1, and 5.2. The only essential difference comes in when we bound the $n+1$ point connectivity function by the product of $n-1$ point and two point connectivity 
functions. (See the proof of Proposition 6.1.) The estimate used here is

$$
\begin{aligned}
& \tau_{p}^{\prime}\left(x_{1}, \ldots, x_{n+1}\right) \leqq \sum_{\substack{\text { pairs } \\
\{i, j) \subset\{1, \ldots, n+1\}}} \operatorname{Prob}_{p} \begin{array}{l}
\left\{\left(x_{1}, \ldots, \hat{x}_{i}, \ldots, \hat{x}_{j}, \ldots, x_{n+1}\right.\right. \text { are con- } \\
\text { nected by a finite cluster }) \circ\left(x_{i}, x_{j}\right. \text { are } \\
\text { connected })\}
\end{array} \\
& \leqq \sum \tau_{p}^{\prime}\left(x_{1}, \ldots, \hat{x}_{i}, \ldots, \hat{x}_{j}, \ldots, x_{n+1}\right) \tau_{p}\left(x_{i}, x_{j}\right),
\end{aligned}
$$

where the final inequality follows from the van den Berg, Fiebig inequality [15].

Remark. Note that, in the above proof, we cannot replace the upper bound for $\tau_{p}^{\prime}\left(x_{1}, \ldots, x_{n+1}\right)$ by

$\operatorname{Prob}_{p}\left\{\left(x_{1}, \ldots, \hat{x}_{i}, \ldots, \hat{x}_{j}, \ldots, x_{n+1}\right.\right.$ are connected by a finite cluster $)$ $\circ\left(x_{i}, x_{j}\right.$ are connected by a finite cluster $\left.)\right\}$.

This is the reason that we are not able to prove the inequality corresponding to (6.4) for the exponents $\Delta_{n}^{\prime}$.

\section{Inequalities for Critical Isotherm Exponents}

In the present section, we briefly describe our final inequalities (1.6) and (1.7) for the critical isotherm exponents.

First we define the percolation system under positive external field $h \geqq 0$. Let us add a "ghost site" $g$ to our lattice $Z^{d}$. We assume that each ghost bond $\{g, x\}, x \in Z^{d}$ is occupied independently with probability $1-e^{-h}$. Then various physical quantities can be defined by regarding the "ghost site" $g$ as "infinity", and replacing the condition $|C(x)|=\infty$ in (2.1)-(2.6) by the new condition $C(x) \ni g$.

When we fix $p$ at its critical value $p_{c}$ and let $h$ approach zero, various critical phenomena are expected to take place. Let us define some critical exponents as the optimal constants satisfying the following relations when $h \downarrow 0$.

$$
\begin{gathered}
\xi\left(p_{c}, h\right) \lesssim h^{-\mu}, \\
M\left(p_{c}, h\right) \lesssim h^{1 / \delta_{1}}, \\
\partial^{n-1} M\left(p_{c}, h\right) / \partial h^{n-1}=\left\langle|C(0)|^{n-1} X(C(0) \in g)\right\rangle_{p, h} \gtrsim h^{-1 / \delta_{n}+1-n}, \quad(n=2,3, \ldots) .
\end{gathered}
$$

Then, by a straightforward modification of the methods in the previous sections, we can easily prove the following.

Proposition 7.1. Whenever $\xi\left(p_{c}, h\right) \nearrow \infty$ as $h \downarrow 0$, the critical exponents $\eta$, $\mu$, and $\delta_{i}$ $(i=1,2,4,6,8, \ldots)$ satisfy

$$
\begin{gathered}
(d-2+\eta) \mu \delta_{1} \geqq 2 \\
d \mu \geqq 1+\left(n / \delta_{1}-1 / \delta_{n}\right) /(n-1) \text { for } n=2,4,6,8,10, \ldots
\end{gathered}
$$


If $M\left(p_{c}, h\right)$ exhibits the following simple power law behavior (as is expected):

$$
M\left(p_{c}, h\right) \sim h^{1 / \delta},
$$

we have $\delta_{l}=\delta$ for any $i$. Then (7.2) reduces to a single inequality

$$
d \mu \geqq 1+(1 / \delta)
$$

\section{Appendix. Extension to Ising Ferromagnets}

In this appendix, we briefly describe the extension of our methods to the Ising ferromagnets.

First let us define Ising model. For an arbitrary positive integer $L$, let $S_{L}$ and $\partial S_{L}$ be

and

$$
S_{L}=\left\{x \in Z^{d}|| x \mid \leqq[L / 2]\right\}
$$

$$
\partial S_{L}=\left\{x \in S_{L}|| x \mid=[L / 2]\right\} .
$$

To each site $x$ in $S_{L}$, we associate a spin variable $\sigma_{x}= \pm 1$. Then the thermal expectation with plus boundary condition is defined by

$$
\begin{aligned}
\langle\ldots\rangle_{L} & =Z_{L}^{-1} \sum_{\sigma_{x}= \pm 1\left(x \in S_{L} \backslash \partial S_{L}\right)}(\ldots) \exp (-H), \\
H & =-(\beta / 2) \sum_{x, y \in S_{L},\|x-y\|_{2}=1} \sigma_{x} \sigma_{y}-h \sum_{x \in S_{L}} \sigma_{x}, \\
\langle 1\rangle_{L} & =1, \quad \sigma_{x}=1 \quad \text { if } \quad x \in \partial S_{L} .
\end{aligned}
$$

We consider the expectation in the infinite volume limit defined by

$$
\langle\ldots\rangle=\lim _{L>\infty}\langle\ldots\rangle_{L}
$$

We define various physical quantities and critical exponents by simply replacing $p$ by $\beta, \tau_{p}(x, y)$ by $\left\langle\sigma_{x} \sigma_{y}\right\rangle, \tau_{p}^{\prime}(x, y)$ by $\left\langle\sigma_{x} ; \sigma_{y}\right\rangle=\left\langle\sigma_{x} \sigma_{y}\right\rangle-\left\langle\sigma_{x}\right\rangle\left\langle\sigma_{y}\right\rangle, M(p)$ by $\left\langle\sigma_{0}\right\rangle$, and $M(p ; L)$ by $\left\langle\sigma_{0}\right\rangle_{L}$ in the definitions in Sects. 2 and 7 . Thanks to the reflection positivity (which is not known for percolation), the correlation length defined as

$$
\zeta(p)^{-1}=-\lim _{|x| \rightarrow \infty} \ln \left\langle\sigma_{0} ; \sigma_{x}\right\rangle /|x|
$$

(limit exists) coincides with that defined by (2.4).

Then by a straightforward extension of the methods described in the text, we can prove

Proposition A.1. The critical exponents of the d-dimensional Ising model satisfy

$$
\begin{gathered}
(d-2+\eta) \delta_{r} \geqq 2, \\
(d-2+\eta) v^{\prime} \geqq 2 \beta,
\end{gathered}
$$




$$
\begin{gathered}
d v^{\prime} \geqq \gamma^{\prime}+2 \beta, \quad d v_{\max } \geqq \gamma+2 \beta, \\
(d-2+\eta) \mu \delta_{1} \geqq 2, \\
d \mu \geqq 1+\left(2 / \delta_{1}\right)-\left(1 / \delta_{2}\right),
\end{gathered}
$$

whenever $\xi(\beta, 0) \nearrow \infty$ as $\beta \backslash \beta_{c}[(\mathrm{~A} .4),(\mathrm{A} .5)]$ or $\xi\left(\beta_{c}, h\right) \nearrow_{\infty}$ as $h \searrow 0[(\mathrm{~A} .6),(\mathrm{A} .7)]$.

As we have mentioned in Sect. 1, most of these inequalities have already been proved by other methods. Our rigorous finite size scaling argument provides a new unified derivation. In particular our proof of the inequality $d v^{\prime} \geqq \gamma^{\prime}+2 \beta$ seems to be simpler than Sokal's highly technical proof [9].

Let us describe how two key arguments in our proof can be stated in the Ising model. Then the rest of the proofs will be just repetitions of those in the main text.

First we discuss the extension of Proposition 4.1 which was the main ingredient of most of our results. Let $L>0$ and $|x|=L$. Define $\langle\ldots\rangle_{h}$ by the formula (A.1) with Hamiltonian $H$ replaced by $H-\tilde{h} \sum_{x \in B} \sigma_{x}$, where $B=\{y|| y \mid=[L / 2]$ or $|x-y|$ $=[L / 2]\}$. Note that when $\tilde{h}=\infty$, the whole system decouples into two finite systems in $L \times \ldots \times L$ cubes and one infinite system. Therefore by the Griffiths II inequality [16], we have

$$
\left\langle\sigma_{0} \sigma_{x}\right\rangle=\left\langle\sigma_{0} \sigma_{x}\right\rangle_{\tilde{h}=0} \leqq\left\langle\sigma_{0} \sigma_{x}\right\rangle_{\tilde{h}=\infty}=\left(\left\langle\sigma_{0}\right\rangle_{L}\right)^{2}
$$

which is nothing other than the desired Ising model version of the inequlaity (4.1).

Next we describe the Ising model counterpart of Lemma 4.1. Let $\Theta_{L}(\beta, h)$ be

$$
\Theta_{L}(\beta, h)=\sum_{y \in \hat{c} S_{L}}\left\langle\sigma_{0} ; \sigma_{x}\right\rangle
$$

Then we have the following.

Lemma A.1. For arbitrary $\beta$ and $h$, we have

$$
\left\langle\sigma_{0}\right\rangle \leqq\left\langle\sigma_{0}\right\rangle_{L} \leqq\left\langle\sigma_{0}\right\rangle+\Theta_{L}(\beta, h)\left\{\left|\ln \Theta_{L}(\beta, h)\right| / 2+3 d L^{d-1} e^{4 d \beta}\right\}
$$

Now a rigorous finite size scaling argument corresponding to Lemma 4.1 can be proved very easily from the above inequalities. If we set $L=\operatorname{const} \xi(\beta, h)|\ln \xi(\beta, h)|$, the upper bound in (A.8) reduces to

$$
\left\langle\sigma_{0}\right\rangle_{L} \leqq\left\langle\sigma_{0}\right\rangle+\operatorname{const} \xi(\beta, h)^{-a}
$$

for sufficiently large $\xi(\beta, h)$. Since the constant $a$ can be made arbitrarily large by choosing suitable constants, (A.9) is sufficient for carrying out our proofs of the critical exponent inequalities.

Remark. Inequality (A.8) also has a consequence on the problem of continuity of the magnetization [17]. By using the fact that $\left\langle\sigma_{0}\right\rangle_{L}$ is a continuous function, we can show that $\left\langle\sigma_{0}\right\rangle$ is continuous in $\beta$ at $\beta=\beta_{0}, h=0$ if $\lim _{L \uparrow} \lim _{\beta \ngtr \beta_{0}} L^{d-1} \Theta_{L}(\beta, h)=0$. 
Proof of Lemma. The first inequality is a simple consequence of Griffiths II inequality. To prove the second inequality, note that

$$
\begin{aligned}
\left\langle\sigma_{0}\right\rangle_{L}-\left\langle\sigma_{0}\right\rangle & =\int_{0}^{\infty} d \tilde{h} \partial\left\langle\sigma_{0}\right\rangle_{\tilde{h}}^{\prime} / \partial \tilde{h}=\int_{0}^{\infty} d \tilde{h} \sum_{x \in \partial S_{L}}\left\langle\sigma_{0} ; \sigma_{x}\right\rangle_{\tilde{h}}^{\prime} \\
& =\int_{0}^{h_{0}} d \tilde{h} \sum_{x \in \hat{\partial} S_{L}}\left\langle\sigma_{0} ; \sigma_{x}\right\rangle_{\tilde{h}}^{\prime}+\int_{h_{0}}^{\infty} d \tilde{h} \sum_{x \in \partial S_{L}}\left\langle\sigma_{0} ; \sigma_{x}\right\rangle_{\tilde{h}}^{\prime},
\end{aligned}
$$

where $\langle\ldots\rangle_{\tilde{h}}^{\prime}$ is defined by the formula (A.1) with Hamiltonian $H$ replaced by $H-\tilde{h} \sum_{x \in \partial S} \sigma_{x} . h_{0}$ is a constant which will be determined later. By the GHS inequality [18], the small field part in the above bound can be bounded as

$$
\int_{0}^{h_{0}} d \tilde{h} \sum_{x \in \partial S_{L}}\left\langle\sigma_{0} ; \sigma_{x}\right\rangle_{\tilde{h}}^{\prime} \leqq \int_{0}^{h_{0}} d \tilde{h} \sum_{x \in \partial S_{L}}\left\langle\sigma_{0} ; \sigma_{x}\right\rangle=h_{0} \Theta(\beta, h) .
$$

In order to bound the large field part, we simply decouple a site $x$ in $\partial S_{L}$ from the rest of the lattice by carefully bounding the local Boltzmann factor. Then tedious but elementary estimates show

$$
\left\langle\sigma_{0} ; \sigma_{x}\right\rangle_{\tilde{h}}^{\prime} \leqq 3 e^{-2 \tilde{h}+4 d \beta}
$$

Combining these results, we get

$$
\int_{0}^{\infty} d \tilde{h} \sum_{x \in \partial S_{L}}\left\langle\sigma_{0} ; \sigma_{x}\right\rangle_{\tilde{h}} \leqq h_{0} \Theta(\beta, h)+2 d L^{d-1}(3 / 2) e^{-2 h_{0}+4 d \beta} .
$$

Setting $h_{0}=|\ln \Theta(\beta, h)| / 2$, we get the desired inequality (A.8).

Acknowledgements. I wish to thank Jennifer Chayes, Lincoln Chayes, and Michael Aizenman for stimulating discussions, Elliot Lieb for continual encouragement and useful comments, and Takashi Hara for helpful remarks.

\section{References}

1. Kesten, H.: Percolation for mathematicians. Boston: Birkhäuser 1982

2. Kesten, H.: Commun. Math. Phys. 109, 109 (1987)

3. Fisher, M.E.: Reports Prog. Phys. 30, 615 (1967)

Baker, G.A.: Phys. Rev. B15, 1552 (1977)

Nickel, B.: Physica 106A, 48 (1981)

Guttmann, A.J.: Phys. Rev. B33, 5089 (1986)

Ito, N., Suzuki, M.: Prog. Theor. Phys. 77 (1987) (in press)

4. Aizenman, M., Newman, C.M.: J. Stat. Phys. 36, 107 (1984); Aizenman, M., Barsky, D., Fernandez, R.: In preparation

Barsky, D.: Rutgers University thesis (1987)

Nguyen, B.G. : Preprint "Gap exponents for percolation processes with triangular condition" 
5. Nguyen, B.G.: J. Stat. Phys. 46,517 (1987)

Chayes, J.T., Chayes, L., Fisher, D., Spencer, T.: Phys. Rev. Lett. 57, 2999 (1987)

Tasaki, H.: Geometric critical exponent inequalities for general random cluster models. J. Stat. Phys. (to appear)

6. Chayes, J.T., Chayes, L.: Commun. Math. Phys. (to appear)

7. Durrett, R., Nguyen, B.G.: Commun. Math. Phys. 99, 253 (1981)

Newman, C.M.: J. Stat. Phys. 45, 359 (1986)

Chayes, J.T., Chayes, L.: Phys. Rev. Lett. 56, 1619 (1986)

8. Aizenman, M., Barsky, D.: Commun. Math. Phys. 108, 489 (1987)

9. Fisher, M.E.: Phys. Rev. 180, 594 (1969)

Liu, L.L., Joseph, R.I., Stanley, H.E.: Phys. Rev. B6, 1963 (1972)

Liu, L.L., Stanley, H.E.: Phys. Rev. B7, 3241 (1973)

Sokal, A.D.: J. Stat. Phys. 25, 25 (1981)

10. Fisher, M.E.: In: Critical phenomena. Green, M.S. (ed), New York: Academic Press 1972

11. Nguyen, B.G.: UCLA thesis (1985)

12. Simon, B.: Commun. Math. Phys. 77, 111 (1980)

13. Aizenman, M.: Private communication

14. van den Berg, J., Kesten, H.: J. Appl. Prob. 22, 556 (1985)

15. van den Berg, J., Fiebig, U.: Ann. Probab. (to appear)

16. Griffiths, R.B.: J. Math. Phys. 8, 478 (1967)

17. Bricmont, J., Lebowitz, J.L.: J. Stat. Phys. 42, 861 (1986)

18. Griffiths, R.B., Hurst, C.A., Sherman, S.: J. Math. Phys. 11, 790 (1970)

Communicated by M. Aizenman

Received May 7, 1987; in revised form June 4, 1987 
